\title{
Stroop interference with response, comparison, and encoding stages in a sentence-picture comparison task*
}

\author{
PHILIP H. K. SEYMOUR \\ The University, Dundee, Scotland
}

\begin{abstract}
The paper discusses a Stroop variant of the word-picture comparison task analyzed by Chase and Clark (1971). The locatives above or below were printed at the center of a reference square. and Ss reported "Yes" if there was a word or an array of Xs in the location specified and "No" if there was not. Lnder the control (Xs) conditions, above displays were classified faster than below displays and true displays faster than false displays. These effects were unaltered when the words "yes" or "no" or "right" or "wrong" were printed in place of the Xs. although there was a small overall increase in response latency under these Stroop conditions that was independent of agreement or disagreement between the distractor and the response to be made. When the locatives up and down were printed on the display, a substantial Stroop effect was observed, and the above-below and true-false effects were eliminated. These results are discussed in relation to theories about the nature of Stroop interference and processes involved in sentence-picture comparisons.
\end{abstract}

Chase and Clark $(1971,1972)$ have discussed a model for sentence-picture comparisons in which it is assumed that meanings of both sentences and pictures may be represented in propositional format in a single abstract or modality-free memory system. The time to indicate the congruence of a simultaneously displayed sentence and picture is thought to be the sum of the durations of sentence and picture encoding operations, a comparison operation, and operations of response selection and execution. These operations are viewed as independent "processing stages," having additive effects on the reaction time (Sternberg. 1969).

The Chase and Clark model was initially applied to analysis of a task in which Ss matched the locatives above or below against the location of a dot that might appear ABOVE or BELOW a reference square (cf. Seymour, 1969). Both Seymour and Chase and Clark reported that above/ABOVE was classified as same faster than below/BELOW and that same displays were classified faster than different displays. Chase and Clark interpreted these results as indicating that "above" concepts were encoded (for both sentences and pictures) more rapidly than "below" concepts and argued that a comparison operation was longer for different than for same displays because a "truth index" had to be altered from "true" to "false" prior to selection of a response.

The experiments to be reported here were initially suggested by the outcome of Experiment 4 in the paper by Chase and Clark (1971). In that experiment. the words "above" and "below" were matched against the location of a dot under conditions of masking of the upper or lower regions of the picture. When the lower region was masked. Ss were instructed to infer present-BELOW from absent-ABOVE. Under this

*This research was supported by the Social Science Research Council of the lnited kingdom under Grant HR:1787/1. Thanks are due Jenn! Greenhalgh for her assistance in obtaining and analyzing the data. condition, reaction times were increased somewhat, but there was no alteration in the magnitude of the effects for above vs below displays or true vs false displays. On the other hand, when the upper region was masked so that Ss inferred present-ABOVE from absent-BELOW, the pattern of the latencies was altered by reduction of the above/ABOVE vs below/BELOW difference and appearance of a substantial delay of below/ABOVE relative to above/BELOW. This altered pattern preserves the effects of above vs below and true vs false and might be expected to occur if the effect of masking was to increase the duration of picture encoding for ABOVE displays, while leaving the true-false and above-below effects unaltered. The difficulty with this explanation is that a comparable delay in encoding of the BELOW location did not occur when the lower region was masked. Chase and Clark proposed, as an answer to this objection, that for unmasked displays Ss might normally attend only to the upper region, defining ABOVE as present-ABOVE and BELOW as absent-ABOVE.

A test of this hypothesis can be made by presenting. in the ABOVE or BELOW location, a stimulus that may interfere with S's response to the display. If Ss normally do not attend to the lower region, an interfering stimulus at the BELOW location will be less disruptive than one at the ABOVE location. A problem then was to find some suitable form of interference. This was resolved by deciding that an irrelevant word could be printed in place of the dot $A B O V E$ or BELOW the reference square, and Ss could be instructed to respond "Yes" if there was a word in the location specified and "No" if there was not. In effect. this defines a Stroop variant of the spatial judgment task.

The introduction of Stroop interference into the sentence-picture comparison task raises questions that are more fundamental than the issue of focal attention to upper or lower regions of a display. In a Stroop task. a naming response to print color. or some other pictorial 
or spatial aspect of a display, may be retarded by presence of an irrelevant word. The degree of interference varies on a semantic gradient, depending on the semantic relatedness of the distractor words to the domain of the naming task (Klein, 1964: Morton, 1969b: Fox, Shor, \& Steinman, 1971). Facilitation effects also occur when there is semantic agreement between the distractor word and the response to be made (Dalrymple-Alford: 1972: Seymour, 1973b). In a sentence-picture matching task. where distinct operations of sentence encoding, picture encoding, comparison, response selection, and response execution are postulated, distractor words may be chosen that are semantically related to one of these supposed stages but not to the others. For example, words having to do with sameness and difference or correctness and incorrectness relate to the comparison or response stages, whereas words referring to spatial location relate to the encoding stages. If, as Chase and Clark have argued, the stages are independent mental operations, the Stroop interference should affect the duration of the stage to which it relates, while leaving the durations of the other stages unaffected.

Previous studies of Stroop interference indicate that the effect occurs when a "between attributes" verbal-pictorial or pictorial-verbal conversion is involved (Treisman \& Fearnley, 1969). Naming ink color is subject to interference from a printed color name (Klein, 1964). Conversely, matching color names to colors may be delayed by the presence of irrelevant incongruous colors (Pritchatt, 1968: Uleman \& Reeves, 1969). These considerations also apply to "same"."different" variants of the Stroop task. "Within attribute" comparisons between ink colors or word identities are not affected by irrelevant verbal or pictorial information (Egeth, Blecker, \& Kamlet, 1969: Treisman \& Fearnley, 1969). However, "between attribute" word-color comparisons are vulnerable to interference from either incongruous words or colors (Treisman \& Fearnley, 1969). "Within attribute" comparisons are subject to interference from words relating to the response $S$ must make to indicate the outcome of his decision. For example, Egeth et al (1969) reported that the vocal responses "same" and "different" were delayed when Ss matched the colors of the print of the words "same" or "different." Dalrymple-Alford and Azkoul (1972) reported that this type of interference occurred only if the distractors were the same as the responses to be made. Thus, Ss responding "Yes" to same print colors and "No" to different colors showed interference from the distractors yes and no but not from the semantically related words right and wrong. This result was reversed for Ss who reported "right" for same and "wrong" for different.

\section{GENERAL METHOD}

Three experiments will be reported, all of which employed the same experimental method as the earlier studies of spatial judgments reported by Seymour (1969) and Chase and Clark (1971). The words "above" or "below" were printed in lowercase Letraset across the midline of a $5 \times 5 \mathrm{~cm}$ outline square. Under a control condition, which was common to all three experiments, a row of three Xs was printed ABOVE or BELOW the square. This defines two same word-picture combinations, above/ABOVE and below/BELOW, and two different combinations, above/BELOW and below/ABOVE. Ss were instructed to respond by calling out "Yes" to same displays and "No" to different displays.

In Experiment 1, the word "yes" or "no" was printed in place of the XS ABOVE or BELOW the square, producing a set of eight additional displays. For four of these, the printed word agreed with the vocal response that $\mathrm{S}$ must make, and for four it disagreed. The cases where "yes" is printed on the above/ABOVE and below/BELOW displays and "no" on the above/BELOW and below/ABOVE displays define a high-compatibility condition. The converse cases. where "no" is printed on the above/ABOVE and below/BELOW displays and "yes" is printed on the above/BELOW and below/ABOVE displays define a low-compatibility condition.

Experiment 2 employed a similar arrangement. in which the words "right" and "wrong" were printed in the ABOVE and BELOW locations. A high-compatibility condition was defined by printing "right" on above/ABOVE and below/BELOW displays and "wrong" on above/BELOW and below/ABOVE displays. In the low-compatibility condition. "wrong" was printed on above/ABOVE and below/BELOW and "right" was printed on above/BELOW and below/ABOVE. In this experiment, therefore, the distractor words were different from the overt responses required ("Yes" or "No") but were semantically related to the notion of correctness or congruence.

In Experiment 3. the locatives up and down were printed on the displays. In the first instance, the displays were classified in terms of semantic agreement or disagreement between the meaning of up or down and the location occupied by the word. Thus, a congruent condition was defined as one in which up was printed in the ABOVE location and down in the BELOW location. An incongruent condition was defined by the cases in which down was printed ABOVE the square and up was printed BELOW the square. Examples of the displays are shown in Fig. 1.

In each experiment, therefore, there was a set of 12 word-picture displays. Four of these belonged to the control (Xs) condition. The eight Stroop displays were divided into a conflict set and an agreement set. depending on the relationship between the meaning of the distractor word and the outcome of response or comparison processes (Experiments 1 and 2) or the operation of location encoding (Experiment 3). In the experiments, the 12 displays were presented in randomized sequences that were independently determined for each S. Ss were instructed to report "Yes" if a word or array of Xs occupied the location specified by above or below and "No" if it did not. Ninety-six observations were taken on each S, making a total of 8 observations per display.

The displays were photographed and mounted as slides for rear projection. The apparatus was set up to provide an auditory warning signal of about $750-\mathrm{msec}$ duration followed. after a l-sec delay, by onset of the display. The displays appeared as a white outline on a dark ground and subtended a visual angle of about 3 deg under the conditions of the experiment. The display appeared when a shutter located close to the light source of a modified Gaf projector was displaced and remained on view for 1 sec. A Venner millisecond stopclock started coincidentally with onset of the display and stopped when the S's vocal "Yes" or "No" response closed the relay of a voice key.

Different groups of eight $\mathrm{Ss}$ served in each of the three experiments. They were all volunteers, drawn from classes at the University of Dundee. Ss were tested individually in a quiet darkened room. 


\section{RESULTS}

The latency data may be examined with respect to the following questions. First. it is necessary to establish whether or not the above-below and true-false effects that define the major stages of the Chase and Clark model for sentence-picture comparisons have been obtained in the present experiments. Second, one may ask whether the Stroop conditions result in reaction times that differ from those obtained under the control conditions and. third. if agreement or conflict between a distractor word and the outcome of a supposed response or encoding process results differentially in facilitation or retardation of the response. In addition, two predictions of the Chase and Clark sentence-picture comparison model can be considered. First, the suggestion of Chase and Clark (1971) that Ss infer BELOW from absent-ABOVE implies that Stroop effects will be greater when the distractor word is ABOVE the square than when it is BELOW it. Second, if sentence encoding and picture encoding are successive and independent operations, occurring in that order, incongruent up and down distractors will add to the duration of a picture encoding operation but will leave differences between above and below and between true and false unaffected. The Chase and Clark model also predicts that yes/no and right/wrong interference will
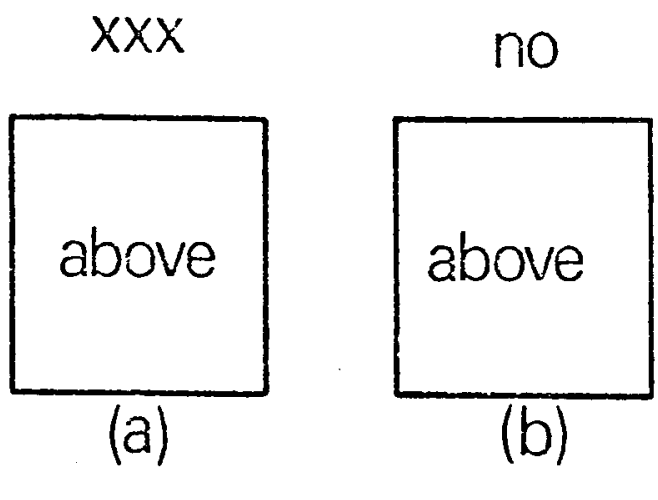

\section{wrong}

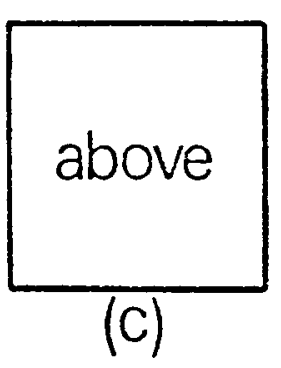

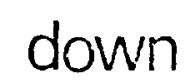

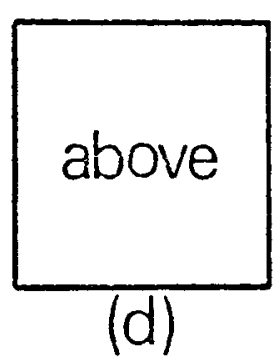

Fig. 1. Examples of the word-picture displays used in the experiments. These are all above/ABOVE displays, classifiable by the report "Yes": (a) control condition. (b) yes no distractors (Experiment 1). (c) right $/ \mathrm{w}$ rong distractors (Experiment 2), and (d) up down distractors (Fxperiment 3).
Table 1

Summary of Latency Data (Milliseconds) for Classification of Above-Below. Word-Picture Displays by the Reports "Yes" or "No" Under the Control Condition and Under Stroop Conditions Where the Distractors Yes or No Might Agree With or Conflict With the Report Required (Experiment 1)

\begin{tabular}{lcrrrr}
\hline & \multicolumn{4}{c}{ Display } \\
\cline { 2 - 5 } & $\begin{array}{l}\text { Above/ } \\
\text { ABOVE }\end{array}$ & $\begin{array}{c}\text { Below/ } \\
\text { BELOW }\end{array}$ & $\begin{array}{c}\text { Above/ } \\
\text { BELOW }\end{array}$ & $\begin{array}{c}\text { Below/ } \\
\text { ABOVE }\end{array}$ & Mean \\
\hline Control (Xs) & 770 & 883 & 949 & 980 & 896 \\
Agree & 824 & 952 & 1009 & 1011 & 949 \\
Conflict & 882 & 932 & 1015 & 995 & 956 \\
\hline
\end{tabular}

not affect the magnitude of the above-below effect. although there may be an effect on the comparison stage, i.e., on the size of the true-false effect.

\section{Experiment 1}

Table 1 presents a summary of the latency data for the yes/no displays and the control (Xs) condition. Inspection suggests that the data reproduce the above-below and true-false effects of the earlier studies and that there is a small Stroop effect which is about equivalent for high-and low-compatibility displays and which is generally independent of the location of the distractor word. An analysis of variance, using a repeated-measures design with conditions (control, high compatibility. low compatibility) and displays (above/ABOVE, below/BELOW, above/BELOW, and below/ABOVE) as factors, indicated that the effect for conditions was not quite significant at the .05 level $(\mathrm{F}=3.3 \mathrm{.} \mathrm{df}=2 / 14)$ but that there was a significant effect for displays $(F=23.47 . \mathrm{df}=3 / 21, \mathrm{p}<.001)$. The interaction of Displays by Conditions was not significant $(\mathrm{F}<1, \mathrm{df}=6 / 42)$. If the Stroop effect was more reliable, this absence of an interaction with displays might be taken as contrary to predictions following from Chase and Clark's (1971) proposal about focal attention to the upper region of the figure. The weakness of the Stroop effect and the lack of differentiation between the high- and low-compatibility conditions appear inconsistent with the findings of Egeth et al (1969) and Dalrymple-Alford and Azkoul (1972) for serial versions of the Stroop task.

The displays effect was evaluated by collapsing the data across conditions and carrying out a repeated-measures analysis in which response ("Yes" vs "No") and words (above vs below) were factors. The difference of 120 msec between "Yes" and "No" response times was highly significant $(F=52.12$. $\mathrm{df}=1 / 7, \mathrm{p}<.001)$. There was also a difference of about $50 \mathrm{msec}$ between above and below displays $(\mathrm{F}=9.57$. $\mathrm{df}=1 / 7 . p<.025)$ that interacted with the response effect $(F=8.05$. $d f=1 / 7 . p<.05)$. The interaction occurred because the above-below effect was almost entirely attributable to the difference of about $100 \mathrm{msec}$ between above ABOVE and halow/BELOW displays. 
Table 2

Summary of Response Latencies (Milliseconds) for Classification of $A$ bove-Below Displays by the Reports "Yes" or "No" Under Control Condition and Under Stroop Conditions When the Distractors Right or Wrong Might Agree or Conflict With the Decision About the Truth or Falsity of the Display (Experiment 2)

\begin{tabular}{lcccrr}
\hline & \multicolumn{4}{c}{ Display } \\
\cline { 2 - 5 } & $\begin{array}{c}\text { Above/ } \\
\text { ABOVE }\end{array}$ & $\begin{array}{c}\text { Below/ } \\
\text { BELOW }\end{array}$ & $\begin{array}{c}\text { Above/ } \\
\text { BELOW }\end{array}$ & $\begin{array}{r}\text { Below/ } \\
\text { ABOVE }\end{array}$ & Mean \\
\hline Control (Xs) & 843 & 918 & 966 & 990 & 929 \\
Agree & 891 & 960 & 1003 & 1002 & 964 \\
Conflict & 918 & 962 & 1013 & 1047 & 984 \\
\hline
\end{tabular}

These results are essentially the same as those obtained in the previous studies and indicate that the above-below and true-false effects are independent of the presence or absence of response-related distractors in the location to be verified.

Errors occurred on $4.2 \%$ of trials in this experiment. Within conditions, the rates were $3.5 \%$ for the control $\mathrm{Xs}, 2.3 \%$ for the high-compatibility condition, and $6.6 \%$ for the low-compatibility condition. Under the latter condition, almost all the errors occurred when "no" was printed on above/ABOVE or below/BELOW displays. There is some indication here, therefore, that the low-compatibility condition resulted in some increase in error rate (cf. Dalrymple-Alford \& Azkoul, 1972), but the number of errors involved is very small, and this aspect of the data was not analyzed statistically for this reason.

\section{Experiment 2}

The latency data for the right/wrong displays have been summarized in Table 2 . In appearance, the data are very similar to the results of Experiment 1 . The introduction of the Stroop conditions caused a slight delay of reaction relative to the control condition, but this effect did not interact with differences among displays and did not differ greatly in magnitude for the high- and low-compatibility conditions. The analysis of variance confirmed that there were highly significant differences among displays $(F=16.4, \quad d f=3 / 21$, $\mathrm{p}<.001)$ and also showed a significant effect for conditions $(F=7.73, \mathrm{df}=2 / 14, \mathrm{p}<.01)$. As in the previous experiment, the Displays by Conditions interaction did not approach significance $(F<1$, $\mathrm{df}=6 / 42)$.

In order to evaluate the displays effect, the data were collapsed across conditions and a test was made on the effects of above vs below and true vs false displays. The overall difference of $40 \mathrm{msec}$ between above and below displays was significant $(F=7.18, \mathrm{df}=1 / 7, \mathrm{p}<.05)$, as was the difference of $89 \mathrm{msec}$ between true and false displays $(\mathrm{F}=32.19, \mathrm{df}=1 / 7, \mathrm{p}<.001)$. In this experiment, the interaction between these factors was not significant $(\mathrm{F}=3.59, \mathrm{df}=1 / 7)$, although the above-below effect was again primarily attributable to the difference of $62 \mathrm{msec}$ between the means for above/ABOVE and below/BELOW combinations.

As in Experiment 1, therefore, response-related Stroop interference did not affect the basic above-below and true-false effects. The significant interference effect that occurred was independent of these distinctions and was also semantically indiscriminate, in that the interference was not dependent on a semantic mismatch between the distractor and the response required. Further, the interference was independent of the location occupied by the distractor and was not greater for the ABOVE than for the BELOW location.

\section{Experiment 3}

In the third experiment, the distractors were the words "up" and "down." These words are related semantically to the concepts "above" and "below," in that they refer to a dimension of verticality and to positive and negative poles on this dimension. In the terminology of Chase and Clark (1971), both above and up might be assigned the representation (+verticality(+polar)), whereas below and down will read (+verticality(-polar)). If, as Chase and Clark have argued, $\mathrm{S}$ compares the sentence and picture by encoding the sentence first and by then encoding the picture in the same abstract format, a locative distractor that is incongruent with respect to the position it occupies might delay the operation of establishing a representation of the picture. In the upper section of Fig. 2, the latency data have been summarized for the control condition and for the cases where the distractors up and down either matched or conflicted with the locations they occupied.

It is obvious that the results in this experiment are very different from those of Experiments 1 and 2, because major Display by Conditions interactions occur. The analysis of variance shows a significant effect for conditions $(\mathrm{F}=4.44, \mathrm{df}=2 / 14, \quad \mathrm{p}<.05)$ and a significant effect for displays $(F=14.22, \mathrm{df}=3 / 21$, $\mathrm{p}<.001)$. The Displays by Conditions interaction is also significant $(F=13.27, \mathrm{df}=6 / 42, \mathrm{p}<.001)$. Additional analyses indicated that the interactions of displays with the control vs conflict conditions or with the agree vs conflict conditions were significant at the .001 level but that the interaction of displays with the control vs agree conditions was not significant.

This result is clearly contrary to the predictions of the Chase and Clark model. If, under the conflict condition, a constant increment was added to the duration of the picture encoding stage, there would be an effect for conditions but no interaction. Inspection of Fig. 2 will suggest that the presence of a conflicting locative resulted in a substantial delay for the same displays, above/ABOVE and below/BELOW, but in some degree of facilitation for the different displays, above/BELOW and below/ABOVE. This implies that the locatives up 
and down are not causing a general increase in the duration of an independent picture encoding operation. Rather, the interference appears to be related to the process of word encoding. This point is demonstrated in the lower panel of Fig. 2. where the data have been plotted with match or mismatch in the meanings of above or below and up or down as a parameter. It is clear from this figure that presentation of above and down or below and up together on the display was sufficient to cause a substantial delay in reaction time and also to eliminate the effects of above vs below and true vs false. When up was presented with above or down with below. the results were identical to those obtained under the control condition, with the exception of "No" responses to below/ABOVE.

The analysis of variance of these data showed significant effects for conditions $(F=36.21 . \mathrm{df}=2 / 14$. $\mathrm{p}<.001$ ) and for displays, and a significant Displays by Conditions interaction $(F=3.6, d f=6 / 42, p<.01)$. Reaction times under the semantic mismatch condition were significantly longer than times under the control condition $(\mathrm{F}=26.2, \mathrm{df}=1 / 7, \mathrm{p}<.01)$, and this effect interacted with the displays effect $(F=4.82, \mathrm{df}=3 / 21$, $p<.025)$. The interaction occurred because there were effects significant at the .01 level for above vs below and true vs false under the control condition, but these effects were both absent under the semantic mismatch condition. where $F(1.7)=1$ for above vs below and 2.27 for true vs false. Reaction times for the semantic match condition did not differ significantly from times for the control condition $(F=2.95, \mathrm{df}=1 / 7)$. However. these conditions interacted with the differences among the word-picture displays $(\mathrm{F}=4.27 . \mathrm{df}=3 / 21 . \mathrm{p}<.025)$. This interaction depended on the difference between above/BELOW and below/ABOVE displays, which was significant at the .025 level under the semantic match condition but which did not occur under the control condition. One consequence of this was that an analysis of the semantic match data, in which above vs below and true vs false were factors. showed nonsignificant effects for both above-below $(\mathrm{F}<1 . \mathrm{df}=1 / 7)$ and true-false $(F=4.14 . d f=1 / 7)$. although there was, of course. an interaction between these factors $(F=20.63 . \mathrm{df}=1 / 7$. $p<.01$ ). Thus, the locatives up and down were ef fective in eliminating the above-below and true-false eftects under both the semantic match and the semantic mismatch conditions.

\section{GENERAL DISCUSSION}

The present paper has attempted a merger of concerns hitherto discussed under the headings of "Stroop effects" on the one hand and "sentence-picture comparisons" on the other. A general intention was to define the locus of Stroop interference by introducing. within a single experimental situation. distractors thit related to the response or encoding stages of the sentence-picture comparison. Dalrymple-Alford and
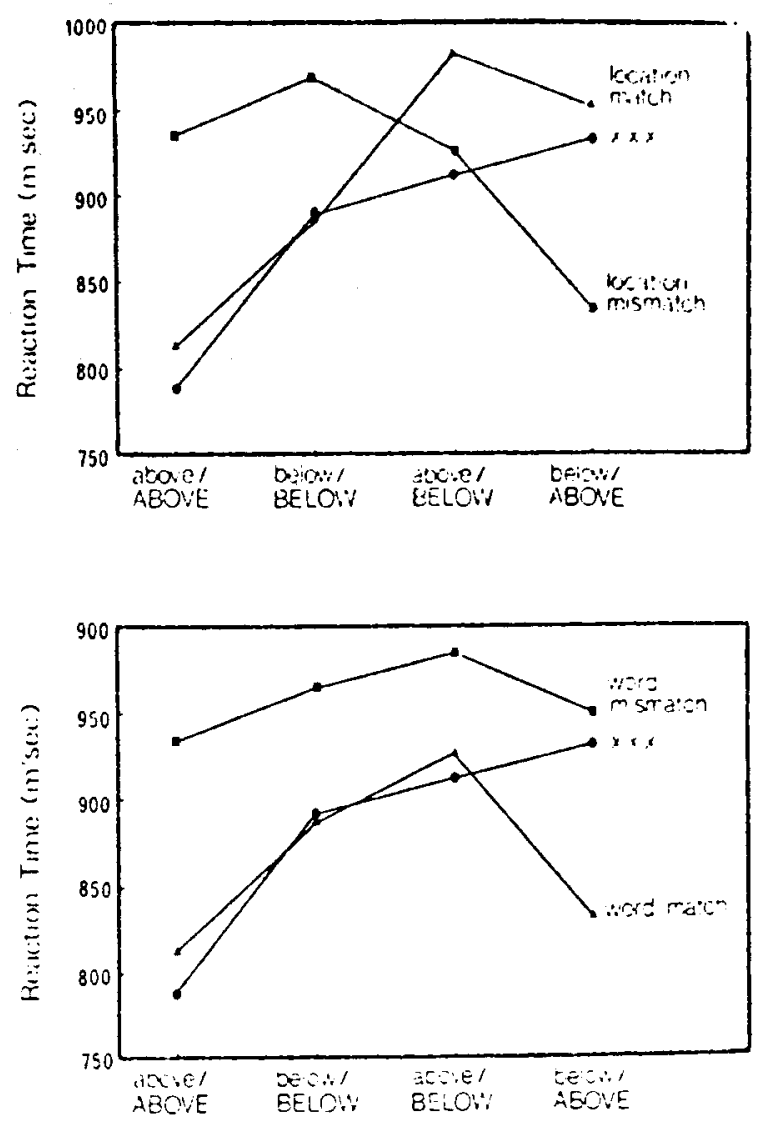

Fig. 2. Summary of latency data for control condition and up/down distractors (Experiment 3). The upper panel shows the data plotted to show the effect of match/mismatch between the meaning of the distractor and the location it occupied. The lower panel shows the same data plotted to show the effect of match/mismatch between the word "above" or "below" and the distractor up or down.

Azkoul (1972) have discussed three hypotheses about the locus of Stroop interference: (1) A response competition theory states that covert responses to the display and to the distractor compete for control over articulation. (2) A response selection explanation states that $\mathrm{S}$ has difficulty in choosing between a response to the display and a response to the distractor. (3) A perceptual theory states that the distractor captures some processing capacity and thus delays the encoding of the display.

The data of Experiments 1 and 2 are difficult to reconcile with either the response competition or the response selection interpretations. If response competition was responsible for the effect. the distractors yes and no should result in a delay of response whenever there was conflict between the identity of the distractor and the vocal "Yes" or "No" response required. In fact the effect of distractors in Experiment 1 was not quite significant. and there was no indication that conflict or agreement between the distractor and the response affected the latency. Similarly. if the effect accurred at the stage of response 
selection and depended on a choice between a semantic representation of the congruence of the display (see Seymour. 1971. for evidence on this point) and of the meaning of the distractor. the data of Experiment 2 would be expected to show greater interference effects when right or wrong conflicted with the outcome of the true-false decision than when it agreed. In practice, the presence of the distractors right and wrong caused an overall delay of response in Experiment 2, but the effect was semantically indiscriminate or independent of conflict or agreement between the distractor and the true-false decision.

In general. therefore, the data of Experiments 1 and 2 may most easily be reconciled with some version of the perceptual theory proposed by Hock and Egeth (1970). This theory states that presence of a distractor adds some constant amount to the time required to encode the display. This could be a matter of threshold adjustments on evidence counters involved in the true-false decision (cf. Bindra, Williams. \& Wise. 1965). Thus. if there were two counters, one for sameness and one for difference. detection of a distractor on the display might result in an equivalent rise in threshold on both counters. This would increase the duration of the operation of sampling display features but would not affect a standing difference in threshold between the true and false counters, differences in time to respond "Yes" vs time to respond "No," or differences in encoding time for above vs below statements.

A theory of this type will encounter difficulty in explaining the semantic gradient effect, unless one is willing to argue that threshold adjustments on the true and false counters depend on some semantic analysis of the distractor. Gradient effects of the type obtained in the studies by Morton (1969b) and Hock and Egeth (1970) could be accommodated if the increment in threshold for both counters was proportional to some index of semantic proximity of distractor to the domain considered at the encoding stage of the comparison. This modification takes account of the observation that Stroop interference in the spatial judgment task is greater for the distractors up and down than for right and wrong or yes and no. It does not, however, explain the interaction of distractors with the above-below and true-false effects in Experiment 3 or the absence of Stroop effects observed under conditions of semantic correspondence between up or down and above or below.

These aspects of the results can be accommodated if certain additional assumptions are made about the effects of preliminary analysis of the display on settings of the thresholds of the true and false counters. Schaeffer and Wallace (1970) described a model for comparison of word meanings which stated that semantic similarity, as determined by a preliminary wholistic comparison of two word concepts, effected differential adjustments in thresholds of counters involved in determining sameness or difference with respect to some specified dimension (e.g.. whether lion and cedar are "both living"). Schaeffer and Wallace argued that semantic similarity lowers the threshold for a same decision and raises the threshold for a different decision. Seymour $(1973 \mathrm{a}, \mathrm{c})$ has proposed a modification of the Schaeffer and Wallace model that incorporates the further assumption that the semantic representation of a word or picture may be predominantly positive or negative, in the sense that above and up are affirmative concepts, having a (+polar) feature: whereas below and down are inherently negative concepts, having the feature (-polar). Positivity, like semantic similarity. tends to lower the threshold for a same decision and raise the threshold for a different decision. Conversely. negativity lowers the threshold for a different decision but may raise the threshold for a same decision.

The model is based on the assumption, therefore, that variations in response latency in a sentence-picture comparison task reflect optional adjustments in the settings of thresholds for production of "Yes" or "No" responses rather than absolute differences in the durations of encoding or comparison stages. This proposal may be formulated in the terms of the logogen model of Morton (1968, 1969a). The logogens corresponding to the vocal responses "Yes" and "No" may normally show a threshold difference that favors the response "Yes." It is this distinction that underlies the true-false effect, rather than the occurrence of an operation of altering a "truth index" from true to false. That this is so is suggested by the observation that true-false effects do not occur in all experimental situations. For example, Seymour (1970) reported that the effect depended on the size of the ensemble of names used in a word-shape comparison task. Further, the effect was eliminated in an above-below task in which Ss were instructed to report "No" for true displays and "Yes" for false displays (Seymour, 1973a). In another study, in which Ss matched above or below against the location of a dot relative to an inverted reference face, the true-false effect was again eliminated (Seymour, 1973c). The presence of the distractors up and down in Experiment 3 also eliminated the true.false difference under both the semantic match and mismatch conditions. Under other circumstances, such as horizontal orientation of the reference face (Seymour, 1973c) or inclusion of yes/no and right/wrong distractors in Experiments 1 and 2, the true-false effect remains unimpaired. Also, it may hold for one condition within an experiment but not for another. Thus, the effect occurs on trials when the reference face is normally oriented but not on trials when the face is inverted (Seymour, 1973c). Similarly, in Experiment 3 the effec $\imath$ was eliminated under the Stroop conditions but maintained under the control condition. These results appear sufficient to demonstrate that the true-false effect does not depend on alteration of a "truth index" but on an optional threshold 
difference that may be modified within the period of preliminary analysis of the display. Thus, the model of the comparison stage proposed by Clark and Chase (1972) appears to be incorrect.

With regard to the above-below difference, the present model provides a compatible alternative to the interpretation proposed by Chase and Clark (1971). If, as Clark has argued, above is an affirmative concept and below an inherently negative concept, the semantic representation of an above/ABOVE display will include a predominance of positive components, whereas the representation of a below/BELOW display will include many negative components. If the positivity of the above/ABOVE display is semantically represented during encoding and these affirmative semantic features are transferred to the logogen system, the threshold for a "Yes" response will be reduced, and some facilitation of the reaction will occur. Some support for an interpretation of this type is given by Seymour's (1973a) finding that the above-below difference is eliminated when above/ABOVE and below/BELOW must be classified by the report "No," as is a comparable difference between large and small (Seymour, 1971). A main point, again, is that an above-below effect appears not to result from an absolute difference in encoding time but to depend on an optional response bias that can be modified by quite small alterations in the experimental procedure. In the study of horizontal and normal or inverted faces, the above-below effect was attenuated when the face was horizontal and eliminated when the face was inverted (Seymour. 1973c), and it was argued that this reflected an increase in the number of negative components in the representation of the picture. Quite similar arguments may be proposed to account for the elimination of the effect under the semantic mismatch condition of Experiment 3. Presentation of down on an above/ABOVE display will involve the introduction of some degree of negativity. just as presentation of up on a below/BELOW display will introduce some positivity. This might be sufficient to counteract the difference in threshold level for the "Yes" logogen associated with normal displays. If a general rise in threshold occurred as a consequence of detection of a distractor on the display (see earlier discussion of Stroop effects in Experiments 1 and 2). or if semantic dissimilarity raised the "Yes" threshold (Schaeffer and Wallace. 1970), the expected outcome would be an increase in reaction time and elimination of the above-below difference. If semantic dissimilarity raises the "Yes" threshold and lowers the "No" threshold, this may also explain why the true-false difference disappeared under the semantic mismatch condition.

An advantage of the optional bias model is that it incorporates provision for facilitation of "No" responses. In the faces study, there was an interaction of face orientation and difference between the displays above/BELOW and below/ABOVE. and in was argued that this depended on an alteration in the relative preponderance of negative components in the representations of these displays (Seymour, 1973c). In Experiment 3, facilitation of "No" responses to below/ABOVE displays occurred under the semantic match condition. This effect is difficult to account for if one accepts Chase and Clark's (1971) assumption of a sequence of independent additive encoding and comparison stages. Indeed, the results for the semantic match condition are quite incompatible with the Chase and Clark model, though intelligible when considered in the context of the optional bias model. Under this condition, facilitation of responses to above/ABOVE displays is not impaired. because semantic similarity and the positivity of up and above will combine to lower the threshold of the "Yes" logogen. A converse effect occurs for the display below/ABOVE, where the negativity of below and down may tend toward reduction of the threshold of the "No" logogen.

One difficulty with this formulation is that, in Experiments 1 and 2, the affirmative distractors yes and right did not facilitate "Yes" responses, nor did the negative distractors no and wrong facilitate "No" responses. As commented earlier, a semantically indiscriminate Stroop effect of the type obtained in Experiment 2 suggests that the distractor was not analyzed semantically. although its presence on the display was registered and resulted in an increase in threshold for both "Yes" and "No" responses. The problem, then, is to explain why the distractors up and down did undergo semantic analysis, i.e., to explain the "semantic power" effect demonstrated by Klein (1964). Following Morton (1969b), one may argue that the experimental task is defined for the $\mathrm{S}$ in terms of requirements for encoding of the display and that interpretation of the task leads to "priming" of some relevant set of logogen units. In the present case, the task requires that $S$ encode spatial features relating to the vertical dimension. Comprehension of this requirement will involve activation of the semantic structure for locatives. with a special emphasis on verticality. Transfer of these features from the semantic memory to the logogen system will prime all locatives, and vertical locatives in particular. Thus, although detection of up and down on the display may result in a general increase in the response thresholds, as suggested for yes/no and right/wrong distractors, this effect will be overridden because up and down gain access to the semantic system by virtue of the primed state of their logogens. This alters the state of the semantic system in terms of semantic similarity. or the balance of positive to negative components. and this affects the response thresholds in the manner described.

These comments are admittedly quite speculative. However, they do indicate that a model based on assumptions justified elsewhere by Morton (1968. 1969a.b) and by Schaeffer and Wallace (1970) can proside an intelligible account of the outcomes of these 
and the other experiments that have been discussed. The advantage of this model over the additive stages model proposed by Chase and Clark (1971) is that it incorporates sufficient flexibility to handle cases where the basic above-below and true-false effects are not found.

\section{REFERENCES}

Bindra. D.. Williams. J. A.. \& Wise. J. S. Judgments of sameness and difference: Experiments on decision time. Science. 1965. 150. 1625-1628.

Chase. W. G.. \& Clark. H. H. Semantics in the perception of verticality. British Journal of Psychology. 1971,62. 311-326.

Chase. W. G.. \& Clark. H. H. Mental operations in the comparison of sentences and pictures. In L. Gregg (Ed.) Cognition in learning and memon. New York: Wiley, 1972.

Dalrymple-Alford. E. C. Associative facilitation and interference in the Stroop color-word task. Perception \& Psychophysics. 1972, 11. 274-276.

Dalrymple-Alford. E. C.. \& Azkoul. J. The locus of interference in the Stroop and related tasks. Perception \& Psychophysics, 1972. 11. 385-388.

Egeth. H. E.. Blecker. D. L.. \& Kamlet. A. S. Verbal interference in a perceptual comparison task. Perception \& Psychophysics. $1969.6 .355-356$

Fox. L. A.. Shor. R. E., \& Steinman. R. J. Semantic gradients and interference in naming color. spatial direction. and numerosity: Journal of Experimental Psychology. 1971. 91. $59-65$.

Hock. H. S., \& Egeth. H. E. Verbal interference with encoding in a perceptual classification task. Journal of Experimental Psychology. 1970, 83, 299-303

Kilein. G. S. Semantic power measured through the interference of words with color naming. American Journal of Psychology. 1964, 77. 576-588.

Morton. J. Grammar and computation in language behavior.
Progress Report No. 6. Center for Research in Language and Language Behavior. University of Michigan. 1968.

Morton. J. Interaction of information in word recognition Psschological Review. 1969a. 76. 165-178.

Morton. I. Categories of interference: Verbal mediation and conflict in card sorting. British Journal of Psychology. 1969b. 60. 329-346.

Pritchatt. D. An investigation into some of the underlying associative verbal processes of the Stroop color effect. Quarterly Journal of Experimental Psychology. 1968. 20, 351-359.

Schaeffer. B.. \& Wallace. R. The comparison of word meanings. Journal of Experimental Psy chology. 1970. 86. 144-152.

Seymour. P. H. K. Response latencies in judgments of spatial location. British Journal of Psychology. 1969, 60. 31-39.

Seymour. P. H. K. Conceptual uncertainty and the latency of judgments of the congruence of word-shape pairs. Acta Psychologica. 1970. 34. 451-461

Seymour. P. H. K. Perceptual and judgmental bias in classification of word-shape displays. Acta Psychologica. 1971. 35. 461-477.

Seymour. P. H. K. Judgments of verticality and response availability. Bulletin of the Psychonomic Society. 1973a. i. 196-198.

Seymour. P. H, K. Stroop interference in naming and verifying spatial locations. Perception \& Psychophysics. 1973b. in press

Seymour. P. H. K. Asymmetries in judgments of verticality. Journal of Experimental Psychology. 1973c. in press.

Sternberg. S. The discovery of processing stages: Extensions of Donders method. Acta Psychologica, 1969. 30. 276-315.

Treisman, A.. \& Fearnley, S. The Stroop test: Selective attention to colors and words. Nature, 1969, 222. 437-439.

Uleman. J. S. \& \& Reeves. J. A reversal of the Stroop interference effect through scanning. Perception \& Psychophysics. 1971, 9 , 293-295.

(Received for publication A pril 8. 1973: accepted May 9. 1973.) 